Ann. Zootech., I976, 25 (2), 213-2I9.

\title{
EFFET DES CONDITIONS D'ÉLEVAGE SUR LE COMPORTEMENT DES GÉNISSES DANS UNE SITUATION DE COMPÉTITION ALIMENTAIRE
}

\author{
Marie-France BOUISSOU et J. H. HÖVELS \\ avec la collaboration technique de Colette Lavenet et P. Orgeur \\ Station de Physiologie de la Reproduction, \\ Centre de Recherches de Tours, I. N.R, A., \\ Nousilly, $3 \% 380$ Monnaie
}

RÉSUMÉ

L'étude a porté sur un troupeau de génisses constitué par la réunion de quatre 1ots d'élevage. Quatre génisses provenant soit d'un même lot d'élevage (3 cas), soit de lots différents (9 cas) ont été mises en compétition pendant io $\mathrm{mn}$ pour un aliment concentré placé dans une auge de i, io $\mathrm{m}$ de long. Une première série d'épreuves $(\mathrm{N}=3)$ a cu lieu deux mois après la réunion des lots d'élevage, une scconde série $(N=3)$ a eu lieu six mois après et une troisième série $(N=3)$ un an après.

Dans tous les cas, les animaux ont mangé significativement plus lorsqu'ils étaient en compétition avec des animaux issus de leur propre lot d'élevage, les dérangements subis étaient moins nombreux ainsi que les interactions agressives.

L'élevage en commun depuis la naissance conduit donc à une grande tolérance même dans une situation de compétition intense ; ceci se retrouve encore un an après la réunion des lots d'élevage en un seul troupeau.

\section{INTRODUCTION}

Les relations de dominance qui existent entre les membres de groupes de Bovins entretenus en stabulation libre ont un certain nombre de conséquences défarorables pour les animaux dominés : en particulier, leur alimentation peut être réduite et fractionnée en courtes périodes, ce qui entraîne une grande hétérogénéité des temps d'alimentation (Bouissou, I964; McPheE, McBride, James, I964; Porzig, ig69; Bosc, Bouissou, Signoret, I968). Ces phénomènes sont d'autant plus nets que la compétition est plus intense : aliment appétent distribué sur une faible longueur d'auge. 
Les situations de compétition alimentaire servent d'ailleurs à mettre en évidence les relations hiérarchiques dans de nombreuses espèces (Bovissou, SignoRET, I970).

Cette technique a également été utilisée chez les Bovins (CANDI,AND, BroomQUIST, I965; BOUIssou, I970) ; lorsque la compétition est maximum, c'est-à-dire lorsqu'un seul animal à la fois peut avoir acrès à l'aliment, le dominé est complètement exclus.

Par ailleurs, à côté des interactions de type agonistique, on observe fréquemment au sein de groupes de Bovins des associations entre certains individus lors des phases d'alimentation ou de repos (Bousssou, 1964) et même, lorsque la taille du troupeau est importante, la formation de sous-groupes (ZIMMERMAN-MULLER, ZEEiB, IG7O; FAVRE, I97I); de tels sous-groupes seraient constitués d'animaux arrivés ensemble dans le troupeau, ou provenant de la même étable.

Lors de l'étude d'un troupeau de seize génisses, constitué par la réunion de quatre groupes de 4 animaux, nous avons pu effectivement mettre en évidence une permanence du "groupe élevage " qui se traduit par une association spatiale, aussi bien en stabulation qu'au pâturage, et par une réduction très inportante de l'agressivité entre ses membres (Bouissou, Hövris, I976).

Au cours de cette étude nous avons cherché à préciser quelles pouvaient être les conséquences de ces phénomènes sur les temps d'alimentation des animaux lors d'une compétition.

\title{
MA'TÉRIEL E'T MÉTHODES
}

\author{
1. Animaux, conditions d'cntretien
}

Le troupeau était constitué de i6 génisses de race Frisonne Pie-Noire provenant de 4 groupes d'élevage différents; elles étaient âgées d'environ 2 ans au début de l'expérience.

Les relations de dominance-soumission existant entre chaque couple possible étaient connues avant le début de l'étude; la hiérarchie du troupeau était complexe alors que celle existant au sein de chaque lot d'élevage était de type linéaire. Un seul changement est intervenu au cours de la période expérimentale : l'antépénultième animal est devenu l'individu oméga du groupe.

Les animaux étaient entretenus en stabulation libre $\left(200 \mathrm{~m}^{2}\right)$; ils recevaient du foin à volonté et une clistribution quotidicnne d'aliment concentré à base de mais ct de luzerne déshyclratés.

\section{2. - Eprewes di compétition alimentaive}

Nous axons mis en compétition 12 groupes de quatre animaux constitués soit d'animaux d'un mème lot d'élevage (épreuves intragroupes : 3 cas ( $\left.{ }^{1}\right)$ ), soit d'une combinaison de quatre animaux provenant chacun d'un lot d'élevage différent (épreuves intergroupes : 9 cas).

Ces neuf combinaisons intergroupes ont été établies de telle sorte que tous les animaux interviennent dans deux ou trois d'entre elles et que le même couple ne se retrouve pas dans plusieurs; ceci s'est cependant produit dans 4 cas sut les 54 possibilités d'interactions duelles existant dans les 9 groupes.

Chacune des combinaisons comportait a la fois des animaux occupant au sein du troupeau

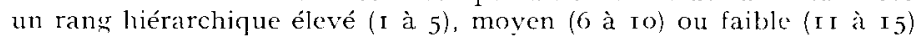

(1) L'ne génisse étant morte accidentellement en cours d'expérience, nous n'avons pas étudié le kt d'élevage dont elle ćtait issue, bien que les trois membres restant aient été utilisés dans les combinaisons interuroupes. 
Chaque groupe a été mis à l'épreuve 9 fois : 3 fois au cours du dcuxième mois suivant la réunion des lots d'élevage en un seul troupeau ( $\mathbf{I}^{\mathrm{re}}$ série), 3 fois 6 mois après ( $2^{\mathrm{e}}$ série), ct 3 fois un an après la réunion des animaux ( $3^{\mathrm{e}}$ série).

Les épreuves de compétition avaient lieu entre I 6 h 30 et $\mathrm{I} 7 \mathrm{~h} 30$, les animaux n'ayant pas eu d'aliment concentré depuis 24 heures. Les quatre animaux étaient sortis du troupeau et introduits dans un parc de $5 \mathrm{~m} \times 6 \mathrm{~m}$; le long d'un des murs se trouvait une auge de $\mathrm{I}$, Io $\mathrm{m}$ de long remplie de l'aliment. Quatre observateurs chronométraient chacun le temps d'accès à l'auge d'un animal, et enregistraient à l'aide d'un compteur manuel le nombre d'interruptions spontanées ou provoquées par une interaction agressive. Les interactions agressives étaient enregistrées sur bande magnétique. Les épreuves duraient Io $\mathrm{mn}$, ce qui représente à peu près la durée normale de consommation de la ration journalière d'aliment concentré. A la fin de l'épreuve les animaux rejoignaient le troupeau. Chaque animal ne prenait part qu'à une compétition par jour.

\section{RÉSULTATS}

Le tableau I résume les résultats obtenus : le temps moyen d'alimentation des animaux est significativement supérieur $(P<0,00 I)$ lorsqu'ils se trouvent en présence d'animaux issus d'un même lot d'élevage (épreuves intragroupes) que lorsqu'ils sont opposés à des animaux provenant de lots différents (épreuves intergroupes), et ceci au cours de chacune des trois séries d'épreuves.

Les durées moyennes d'une période de prise alimentaire sont respectivement de 235,224 et 254 secondes lors des épreuves intragroupes alors qu'elles ne sont que de 82,85 et 84 secondes lors des épreuves intergroupes (différences significatives au niveau $0,00 r$ ) ; les animaux sont donc beaucoup plus fréquemment dérangés dans le second cas.

Enfin, le nombre moyen d'interactions agressives enregistrées au cours des épreuves est toujours plus important lors des compétitions intergroupes; la différence est hautement significative pour la première série d'épreuves, le niveau de signification n'est pas atteint au cours des séries suivantes en raison en particulier de la grande variabilité du niveau d'agressivité des différentes combinaisons de quatre animaux réalisées.

Les résultats de chaque animal montrent, qu'à de rares exceptions près, le temps d'alimentation d'une génisse est supérieur lors des compétitions intragroupes. Cependant certains animaux mangent pendant un temps sensiblement égal dans les deux types d'épreuves, ou même plus lors des compétitions intergroupes ; il s'agit soit de l'animal dominant du troupeau, soit des animaux qui, dominés dans leur propre groupe d'élevage, ont un rang moyen plus élevé dans les combinaisons intergroupes. Dans les autres cas, la réduction du temps d'alimentation lors des épreuves intergroupes est d'autant plus forte que les animaux sont situés bas dans la hiérarchie du troupean. Le coefficient de corrélation par rang ( $r$ de SpEARman, in Siegel, I956) entre la réduction du temps d'accès à l'auge et le rang hiérarchique est de $+0,62$ $(\mathrm{P}<0,05)$ lors de la première série d'épreuves; il est également positif mais n'atteint pas le niveau de signification $(r s=+0,33$ et $r s=+0,36)$ lors des autres séries.

Au cours de la première série d'épreuves, les temps moyens d'alimentation sont voisins au sein des lots d'élevage : la différence entre l'animal qui s'alimente le plus et celui qui s'alimente 1 e moins est en moyenne de 2 Io sec. $\pm 6_{5}$; il n'y a pas de corrélation entre le rang social au sein du troupeau et le temps d'alimentation. Par 
contre dans les combinaisons intergroupes, la différence entre les temps extrêmes est en moyenne de $42 \mathrm{I}$ sec. \pm 28 ; elle est significativement supérieure $(\mathrm{P}<0,005)$ à la différence moyenne intragroupes. La corrélation entre rang social et temps d'alimentation est, dans ce cas, hautement significative.

\section{TABLEAU I}

Temps d'alimentation, durée d'une période de prise alimentaire, et nombre d'interactions agressives lors d'épreuves de compítition de $10 \mathrm{mn}$ entre 4 génisses d'un même lot d'élevage (Épreuves intragroupes) ou de lots différents (Épreuves intergroupes)

\begin{tabular}{|c|c|c|c|}
\hline & $\begin{array}{l}\text { Épreuves } \\
\text { intragroupes }\end{array}$ & $\begin{array}{l}\text { Épreuves } \\
\text { intergroupes }\end{array}$ & $\begin{array}{c}\text { Signification } \\
\text { de la } \\
\text { différence }\end{array}$ \\
\hline \multicolumn{4}{|c|}{ 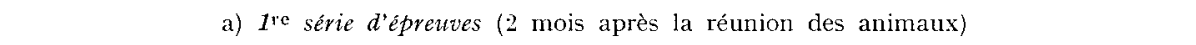 } \\
\hline $\begin{array}{c}\text { Temps moyen (sec.) d'alimentation } \\
\text { par animal }\end{array}$ & $517 \pm 21\left(^{1}\right)$ & $295 \pm 20$ & $\mathrm{P}<0,001$ \\
\hline $\begin{array}{c}\text { Durée moyenne } \\
\text { d'une période de prise alimentaire }(\mathrm{sec} \text { ) }\end{array}$ & $235 \pm 35$ & $82 \pm 12$ & $P<0,001$ \\
\hline $\begin{array}{c}\text { Nombre moyen d'interactions agressives } \\
\text { par épreuve }\end{array}$ & $9,1 \pm 2,2$ & $16,7 \pm 2,0$ & $P<0,01$. \\
\hline
\end{tabular}

b) 2e série d'épreuves (6 mois après la réunion des animaux)

\begin{tabular}{c|c|c|c}
$\begin{array}{c}\text { Temps moyen (sec.) d'alimentation } \\
\text { par animal }\end{array}$ & $407 \pm 36$ & $253 \pm 2.2$ & $\mathrm{P}<0,001$ \\
$\begin{array}{c}\text { Durée moyenne } \\
\text { d'une période de prise alimentaire (sec.) }\end{array}$ & $22 \pm 41$ & $85 \pm 15$ & $\mathrm{P}<0,001$ \\
\hdashline $\begin{array}{c}\text { Nombre moyen d'interactions agressives } \\
\text { par épreuve }\end{array}$ & $12,5 \pm 3,6$ & $\mathbf{1 5 , 6} \pm 2,1$ & $\mathrm{NS}$ \\
\hline
\end{tabular}

c) 3e série d'éprentes (1 an après la réunion des animaux)

\begin{tabular}{|c|c|c|c|}
\hline $\begin{array}{c}\text { Temps moyen (sec.) d'alimentation } \\
\text { par animal }\end{array}$ & $399 \pm 40$ & $262 \pm 22$ & $P<0,001$ \\
\hline $\begin{array}{l}\text { Durée moyenne } \\
\text { d'une période de prise alimentaire (sec.) }\end{array}$ & $25^{\prime}+45$ & $84 \pm 15$ & $\mathrm{P}<0,001$ \\
\hline $\begin{array}{c}\text { Nombre moyen d'interactions agressives } \\
\text { par épreuve }\end{array}$ & $5,8 \pm 2,0$ & $15,5 \pm 2,6$ & NS \\
\hline
\end{tabular}

(1) Ecart-type de la moyenne.

Ultérieurement 1'homogénéité des temps d'alimentation reste supérieure lors des compétitions intragroupes bien que les différences ne soient pas statistiquement significatives. L'absence de corrélation entre rang hiérarchique et temps d'alimen- 
tation intragroupes persiste encore 6 mois après le regroupement des animaux. Cependant I an après, une corrélation positive significative $(\mathrm{P}<0,05)$ existe dans les deux cas aussi bien dans les épreuves intragroupes qu'intergroupes.

\section{DISCUSSION E'T CONCLUSION}

Les résultats de cette étude montrent très nettement l'avantage apporté par la situation "intragroupes ", dans laquelle les animaux ont été élevés ensemble, par rapport à la situation "intergroupes " dans laquelle, bien que se connaissant et même vivant ensemble depuis un an, ils n'ont pas été élevés ensemble.

Lorsque 4 animaux issus d'un mêne groupe d'élevage sont mis en compétition, leur temps d'alimentation moyen est nettement supérieur à ce qu'il est lorsqu'ils sont en compétition avec des animaux provenant de groupes différents; le nombre d'interruptions par animal et le nombre d'interactions agressives survenant au cours de l'épreuve sont plus faibles.

Dans nos conditions expérimentales la compétition était très importante puisque chaque génisse ne disposait que d'environ $30 \mathrm{~cm}$ de longueur d'auge. Néanmoins les quatre animaux d'un même lot d'élevage parvenaient à s'y alimenter simultanément (au moins lors des deux premières séries d'épreuves), alors que pratiquement dans aucun cas il n'y avait plus de deux animaux en même temps à l'auge lors de compétitions intergroupes.

Au cours de la troisième série d'épreuves intragroupes, on retrouve une corrélation entre rang hiérarchique et temps d'alimentation et, dans un certain nombre de cas, l'exclusion de l'animal dominé ; ceci peut s'expliquer par le fait que lors de ces épreuves, qui ont eu lieu un an après les premières, la compétition était encore plus intense ; en effet le poids moyen des animaux est passé de 405 à $530 \mathrm{~kg}$ et il leur était matériellement impossible d'avoir accès à l'auge simultanément. Néanmoins, même à ce moment, les temps moyens d'alimentation sont significativement supérieurs à ce qu'ils sont lors des épreuves intergroupes.

La grande tolérance mutuelle des animaux issus d'un même lot d'élevage a donc des effets à long terme puisqu'elle est encore sensible un an après leur réunion avec d'autres animaux; par ailleurs, une année de contact semble insuffisante pour obtenir ce résultat tout au moins lorsqu'elle se situe entre I 8 et 30 mois. On peut se demander s'il n'existe pas ce que l'on pourrait qualifier de "période critique " pour la création de ce type de relations.

L'importance pratique de ces résultats est évidente : on a en effet montré que les animaux situés au bas de l'échelle sociale subissent un véritable stress que les méthodes modernes d'élevage tendent à augmenter. L'accroissement d'agressivité qui résulte en particulier de la réduction de surface, du remaniement des lots etc. a des conséquences néfastes sur les performances des animaux : réduction de la vitesse de croissance, augmentation de l'indice de consommation, chute de production laitière.

Par ailleurs la constitution de groupes de plus en plus importants ne permet plus de s'attacher aux cas particuliers et oblige à ne prendre en considération que les résultats moyens. Il est donc nécessaire de chercher à minimiser les conséquences 
défavorables de l'existence des relations hiérarchiques pour les animaux dominés, et à avoir une homogénéité maximum.

Nos résultats ont montré la faible agressivité et la tolérance mutuelle des animaux élevés ensemble. Nous avons peut-être là une méthode permettant de réduire l'agressivité intragroupes, d'effacer en partie l'effet défavorable des situations de compétition et vraisemblablement de réduire le stress subi.

A la lumière de ces résultats, le maintien des animaux au sein d'un même groupe depuis le jeune âge jusqu'à l'abattage, pourrait être envisagé, en particulier pour les lots d'engraissement; si les différentes améliorations apportées par ce type de gestion se confirment et s'avèrent suffisantes pour justifier l'acceptation d'autres contraintes alors nécessairement imposées, il pourrait éventuellement être étendu à d'autres situations.

Reçu pour publication en décembre 1975.

\title{
REMERCIEMEN'TS
}

Nous exprimons notre gratitude à toutes les personnes qui nous ont conseillés pour l'analyse statistique des résultats et en particulier Mrs H. Durup et J. Mallard. L'analyse des résultats a été effectuée à la station de biométrie de Nancy; nous remercions les personnes qui y ont participé.

\author{
SUMMARY \\ EFFECT OF REARING CONDITIONS ON THE BEHAVIOUR OF HEIFERS \\ IN A HOOD COMPETITIVE SITUATION
}

This study was made with a herd of 15 heifers constituted by the reunion of 4 different rearing groups.

Four animals competed for food in a through of $1.10 \mathrm{~m}$ length.

The criteria recorded were :

- feeding time of each animal (over ro min),

- number of retreats from the through,

- number of aggressive interactions occuring during the test.

Two situations were studied :

- Intragroup competitions using 4 animals of the same rcaring group (3 cases).

-- Intergroup competitions using 4 animals one from each group (9 cascs).

After the animals from the different groups were brought together, 3 series of tests were carried out in each situation : the first one after two months, the second one after six months and the third one after one year.

In all cases, the animals ate significantly more when they were in competition with animals of their own group; there were less disturbance and aggressive interactions.

Thus, when the heifers had been kept together from birth, they were highly tolerant cven in a situation of severe competition. This phenomenon could still be observed one year after the establishment of the herd. 


\section{RÉFÉRENCES BIBLIOGRAPHIQUES}

Hosc M. J., Bocissou M. F., Signoret J. P., 1968. Conséquences de la hiérarchie sociale sur le comportement alimentaire des bovins domestiques, $93 \mathrm{e}$ Congrès des Sociétés Savantes, Section des Sciences, Tours.

Bocissor M. F., 1964. Observations sur la hiérarchie sociale chez les bovins domestiques. Diplôme d'Études Supérieures. Faculté des Sciences, Paris (non publié).

Botissor M. I*, I970. Technique de mise en évidence des relations hiérarchiques dans un groupe de bovins domestiques. Rev. Comp. Anim., 4, 66-69.

Hocissot M. F., Hövels J. H., 1976. Effet d'un contact précoce sur quelques aspects du compor. tement des bovins domestiques. Biologie du comportement, $\mathbf{n}^{0}$ I (sous presse).

Horissoc M. F., Signoret J. P., i97o. La hiérarchie sociale chez les mammifères. Rev. Comp. anim., 4 43-6I.

Cacoland D. K., Bloomelist D. W., I965. Interspecies comparison of the reliability of dominance orders. J. Comp. Physiol. Psychol., 59, 135-137.

livre Y., I97I. Comportement des bovins en Alpage. Rapport I.N.E.R.M. (non publié).

McPhe: C. P., McBride G., James J. W., I964. Social behaviour of domestic animals. III. Steers in small yards. Anim. Prod., 6, 9-I5.

l'orzig F., r969. Das Verhalten Landwirtschaftlicher Nutztiere. Veb Deutscher Landwirtschaftsverlag. IBerlin.

SIEGEL S., I956. Nonparametric statistics for the behavioral Sciences. McGRaw Hill Book Compagny.

Zinmermax-Mtllek M., Zеeв K., I97i. Sozialstruktur und Aktivität bei Milchkühen. Ter Tierzilchter, 9, 25I-253. 